\title{
Ethical Al in the re-ablement of older people: Opportunities and challenges
}

\author{
Marinka Lanne $\mathrm{DSc}^{\mathrm{a}, *}$, Jaana Leikas $\mathrm{PhD}^{\mathrm{a}}$ \\ ${ }^{a}$ VTT Technical Research Centre of Finland Ltd, Tampere, Finland; *Corresponding author: \\ marinka.lanne@vtt.fi
}

\begin{abstract}
Background: Artificial intelligence (AI) has enormous potential that can be harnessed to promote the health, well-being, safety, and quality of life of older people as well as the work of rehabilitators. Discussion on its utilisation in rehabilitation and therapy work has gradually begun. Our study looks at homecare and re-ablement of older people, where many new technologies are already actively used, but the debate over the use of Al is still limited.

Objective: Both the nature of $\mathrm{Al}$ and the application of its use, especially in situations involving vulnerable people, raise ethical issues in the debate. We discuss opportunities and challenges that arise when applying $\mathrm{Al}$ to rehabilitation processes and particularly to occupational therapy. The aim is to shed light on how Al can be implemented in an ethically sustainable way. As re-ablement services usually employ occupational therapists as members of the rehabilitation team for older people, we chose the Occupational Therapy Intervention Process Model as an example of a customer-oriented process model for the rehabilitation implementation.

Method: Based on the content analysis of nine interviews and 22 scientific articles, perceptions of opportunities and challenges related to Al utilisation were studied and the results were linked to the occupational therapy intervention process.

Results: Opportunities enabled by AI for rehabilitation were identified, and they include clinical decision support, workload optimisation, intervention planning, remote monitoring of home rehabilitation, and the use of Al-based rehabilitation. Most of the Al challenges related to social trust and the experience of autonomy, power structures, privacy concerns, data security, transparency, and biases leading to unfair treatment of individuals and patient groups.

Conclusion: Creating new competence, building and maintaining the trust, and leading the cultural change were considered key issues in Al utilisation. Ethics guidelines for trustworthy $\mathrm{Al}$ set out the key ethical principles, but leave much room for interpretation for the implementation of ethical $\mathrm{Al}$ in rehabilitation. Deliberation of the ethical impacts of $\mathrm{AI}$ requires discussion of values and attitudes, broad citizen involvement, motivation, training for both professionals and citizens, knowledge building through trusted networks, and multi-professional collaboration.
\end{abstract}

Keywords: artificial intelligence, ethics, older people, re-ablement, occupational therapy

\begin{abstract}
INTRODUCTION
Re-ablement is a particular approach within home care and rehabilitation of older people. It supports older adults in developing confidence and relearning self-care skills, thereby increasing their independence. Home care re-ablement services consist of personal care and help with activities of daily living in such a way as to enable older people to develop both the confidence and practical skills to carry out these activities themselves. The provision of supportive technology is an important feature of home care re-ablement services (e.g. Bond et al, 2015). Overall, the aim is to maximise long-term independence and quality of life (Glendinning et al., 2010; Petch, 2008).
\end{abstract}

The main goal in re-ablement is to promote customers' independence, meaningful occupation and functional abilities to fulfil their daily routines and roles. Many different social and health services are working towards this goal. The perspective is most emphasized in the field of occupational therapy (OT). Nowadays, the increasing integration of technology into customers' daily lives, as well as into therapists' workdays, is raising the issue of changes in the profession. In addition to many kinds of applications and patient record systems, telehealth solutions, sensor technology, etc., artificial intelligence (AI) is also changing the world by permeating society as a whole. From research and theoretical reflection that began in the 1950s, the study of Al has progressed to practical applications, and the healthcare sector is one of the important applicators that is willing to benefit from Al. 
The application of AI will have significant potential in social and health care and rehabilitation over the next few decades. Al projects in those fields are now advancing at a rapid pace. Currently, $\mathrm{Al}$ is utilised in data analysis, data storage, assessment of care needs, diagnosis, and rehabilitation interventions. Al has made progress, and it already brings benefits in many areas, such as in the treatment of sleep disorders, oncology, cardiology, surgery (robots), and the research and treatment of lung diseases. Al applications supporting diagnosis use an extensive amount of patient and research data and, typically, methods of image recognition and deep learning. The benefits include additional information, refinement, and enhancement to support the diagnosis made by a clinical doctor (Tuominen, 2019).

Although technology is not a substitute for a therapist, Al can be used to support action. Naturally, certain limitations must be taken into account. As Professor Lili Liu (2018) states, a customer-centric approach, the therapeutic use of self, critical thinking and the creative problem-solving of e.g., occupational therapists make it difficult to replace the profession with any automation. In addition, teaching an $\mathrm{Al}$ algorithm human qualities and experiences, tacit knowledge and cultural contents is difficult - perhaps even impossible — in light of current developments. Still, Al may offer some benefits for the rehabilitation process, e.g. by analysing large amounts of data.

Despite the obvious potential and benefits of $\mathrm{Al}$ (and related evolving technologies), careful consideration of $\mathrm{Al}^{\prime}$ 's short- and long-term impacts on individuals and societies, and humanity in general, is needed. For this reason, the High-Level Expert Group on Artificial Intelligence (established by the European Commission) has drafted 'Ethics guidelines for trustworthy $\mathrm{Al}^{\prime}$ (2019). The guidance is focused on fostering and securing ethical and robust $\mathrm{Al}$, and it sets out four ethical principles: respect for human autonomy, prevention of harm, fairness, and explicability.

Our case country, Finland, is ranked fifth globally on the Government Al Readiness Index (2019) after Singapore, the US, the UK, and Germany. In Finland, the National Artificial Intelligence Programme AuroraAI (AuroraAI 2019) is currently striving for the implementation of an operations model for helping citizens and companies to use services in a timely and ethically sustainable manner. The AuroraAl network and the core technology under development directly enable the formation of cross-sectoral service ecosystems for different situations and events in people's lives. The AuroraAl service model is directed at organisations in various sectors of society to support human-centric activities and knowledge-based management. The roles of AI inside the service process should, however, be carefully considered.

In this paper, we have an ethics focus. More precisely, the aim is to shed light on how Al can be implemented in an ethically sustainable way in the field of home care and re-ablement of older people. Human agency is a key issue for reflection in the context of $\mathrm{Al}$ as well as in the re-ablement of older people. For this reason, the OT perspective, which emphasizes a meaningful occupation and customer independence, has been chosen as the most appropriate framework for our purpose. First, we concentrate on ethical principles and requirements for $\mathrm{Al}$ and a professional ethics approach to occupational therapy. Then, we describe the data and methods of our study and discuss the perceptions in terms of the opportunities and challenges of Al. Finally, we conclude with some key issues in Al utilisation and suggest some actions to support therapists in the planning of ethical $\mathrm{Al}$ implementation.

\section{ETHICAL VALUES, PRINCIPLES, AND REQUIREMENTS FOR AI}

In this article, the multidimensional concept of AI is considered as a system utilising different methods, technologies and disciplines. With the help of machine learning algorithms, $\mathrm{Al}$ is taught to simulate human activity, produce rationality and automate analysis, for example (e.g. Russell and Norvig, 2021; Rajkomar, Dean \& Kohane, 2019). $\mathrm{Al}$ is considered here from the perspective of 'narrow $\mathrm{Al}^{\prime}$ supporting human activity rather than displacing it (e.g. Maddox, Rumsfeld \& Payne, 2019). At some level, the wide impact of Al on society has also been taken into account in the reflection of the results. The focus is, however, on the opportunities and challenges of Al when it intertwines with a human-oriented therapy process.

The application of Al will not only change the content of care work but also change professions and jobs and will have potentially extensive economic impacts. This means changes in societal structures and practices. Consequently, Al technologies give rise to many as-yet-unseen ethical issues (Kitchin, 2017, 18). These are associated with the data mass that intelligent systems will collect and share, and concerns regarding security, privacy, confidentiality, data protection, prevention of harm, and informed consent, for example.

The impact of technology can be assessed against a number of ethical principles that are considered universal ethical values (Leikas et al., 2020). In terms of ethics of $\mathrm{Al}$, many public, private and civil organisations and expert groups have introduced visions for designing ethical technology and ethical Al. The most prominent ones are probably the guidelines of the European 
Group on Ethics in Science and New Technologies (EGE, 2018), the European Commission's High-Level Expert Group on Artificial Intelligence (AI HLEG, 2019), and AI4People (Floridi et al., 2018), to mention but a few.

For example, the Al4People's project (2018) (Floridi et al., 2018) has subsumed ethical principles under four overarching principles. These include beneficence, non-maleficence, autonomy (defined as self-determination and choice of individuals), justice (defined as fair and equitable treatment for all), and explicability. The latter is also taken up by many experts, and refers to the concept of 'explainable $\mathrm{Al}^{\prime}$ coined by the European Commission (AI HLEG, 2019). The concept is meant to promote understanding of the functioning of Al systems and the decisions generated as a result of those functions. Explainability captures the need for accountability and transparency. It is necessary for building and maintaining citizens' trust and is the precondition for achieving informed consent from individuals.

\section{AN ETHICAL APPROACH tO Al IN REHABILITATION AND RE-ABLEMENT}

Ethical issues related to the usage of $\mathrm{Al}$ in rehabilitation and re-ablement of older people arise regarding the introduction and adoption of technology rather than the inherent characteristics . We easily fall into looking for ethical problems related to the essence of $\mathrm{Al}$, when we should be asking how $\mathrm{Al}$ could be used to improve the customer's quality of life. Consideration should be given to how technology can help in perceiving and improving the elements of a good life. This is the meaningfulness of technology (Leikas et al., 2020). Ethical issues in re-ablement can be perceived on three levels. Firstly, they concern about good rehabilitation practices and the rights of the customers. Secondly, ethics relate to the foreseen impacts of rehabilitation therapy: what kind of possible impacts can the therapy have, and what is the role of Al in it? Thirdly, there are ethical implications concerning the use and ownership of stored personal data. The ethical issues, in this case, are linked to trust, privacy, and data protection.

In occupational therapy, the ethical usage of $\mathrm{Al}$ has to be aligned with the fundamental values of OT and the corresponding and recognised ethical principles. In this study, the opportunities and challenges of $\mathrm{Al}$ were examined from the perspective of the occupational therapy process and in terms of 'evidence-based' and 'customer-centric' practice. For this purpose, we chose the Occupational Therapy Intervention Process Model (OTIPM), which is a customer-oriented process model for the phased implementation of OT. Against the background, there is also the Transactional Model of Occupation (TMO), where occupational performance, experience, and participation are shaped in the field of influence of various internal and external elements (Fisher \& Marterella, 2019).

An ethical code of conduct and central principles for OT can be formed as follows, adopted (with small modifications) by the Occupational Therapy Code of Ethics of the American Occupational Therapy Association (AOTA). These include:

1. Beneficence. OT personnel shall demonstrate a concern for the well-being and safety of customers. 2. Non-maleficence. OT personnel shall refrain from actions that cause harm.

3. Autonomy. OT personnel shall respect the right of the individual to self-determination, privacy, confidentiality, and consent.

4. Justice. OT personnel shall promote fairness, equity, inclusion, and objectivity in the provision of OT services.

5. Truthfulness. OT personnel shall provide comprehensive, accurate, and objective information when representing the profession.

6. Fidelity. OT personnel shall treat customers, colleagues, and other professionals with respect, fairness, discretion, and integrity. (AOTA, 2020)

Without denying the relevance of all the abovementioned principles, evidence-based and customer-centric approaches bring the key principles together effectively, and therefore we are focusing here on those. Principles $1-3$ and 6 can be merged to customer-centric practice, and principles 4-5 can be combined with evidencebased practice. Customer-centric practice requires therapeutic rapport and a collaborative relationship between the customer and therapist. Respect for customers' perspectives, preferences, values, and choices are highly important (Fisher \& Marterella, 2019). An evidence-based approach here refers to using methods and practising OT in the best possible way: 'doing the right things right' (Alvernik \& Linddahl, 2011). According to Alvernik and Linddahl (2011), the most referenced definition for evidence-based medicine is formulated by Sackett and others (1996): 'the conscientious, explicit and judicious use of current best evidence in making decisions about the care of individual clients'.

\section{Method}

The main opportunities and challenges related to $\mathrm{Al}$ in rehabilitation were identified based on the interview data and a literature review.

\section{Data collection}

In the spring of 2020, we interviewed nine specialists familiar with $\mathrm{Al}$ development and the planning of $\mathrm{Al}$ implementation, and who were currently working in the social and health sec- 
Table 1. Overview of the organisations represented in the interviews

\begin{tabular}{lccc}
\hline Id & Organisation & $\begin{array}{c}\text { Duration } \\
(\mathrm{min})\end{array}$ & $\begin{array}{c}\text { Length of } \\
\text { transcription (pages) }\end{array}$ \\
\hline $\mathrm{A}$ & Local authority (over 200,000 inhabitants) & 72 & 11 \\
$\mathrm{~B}$ & Research and development institute & 88 & 14 \\
$\mathrm{C}$ & Government department & 54 & 8 \\
$\mathrm{D}$ & Government department & 54 & 9 \\
$\mathrm{E}$ & Health technology company & 33 & 6 \\
$\mathrm{~F}$ & Central hospital & 99 & 12 \\
$\mathrm{G}$ & Social insurance institution & 63 & 9 \\
$\mathrm{H}$ & Local authority (under 50,000 inhabitants) & 81 & 13 \\
I & Industry association & 89 & 14 \\
& In total & $\mathbf{6 3 3}$ & $\mathbf{9 6}$ \\
\hline
\end{tabular}

All the interviewees first read the description of the project and the data protection notice, and had the opportunity to ask questions. They signed a form for voluntary consent to participate in the interview. In the following, quotations are used to selectively depict specific aspects raised by the interviewees (Clark \& Gerrig, 1990; Wade \& Clark, 1993). In addition, summative descriptions are used to express opportunities and challenges briefly (Table 3 ).

The qualitative data from the interviews were supplemented by a limited version of an integrated literature review. The synthesis of the literature review was carried out as an integrative review, including a critical review (Birmingham 2000, 33-34) and the steps of a systematic review: layout of the research problem, data acquisition, evaluation, analysis, and interpretation and presentation of results (Cooper, 1989, 15).

After the test searches, a literature search was carried out on the multidisciplinary database ProQuest Central. The search was narrowed down to include the word pair 'artificial intelligence' $^{\prime}$ in the title of the peer-reviewed scientific

\section{Interview data}
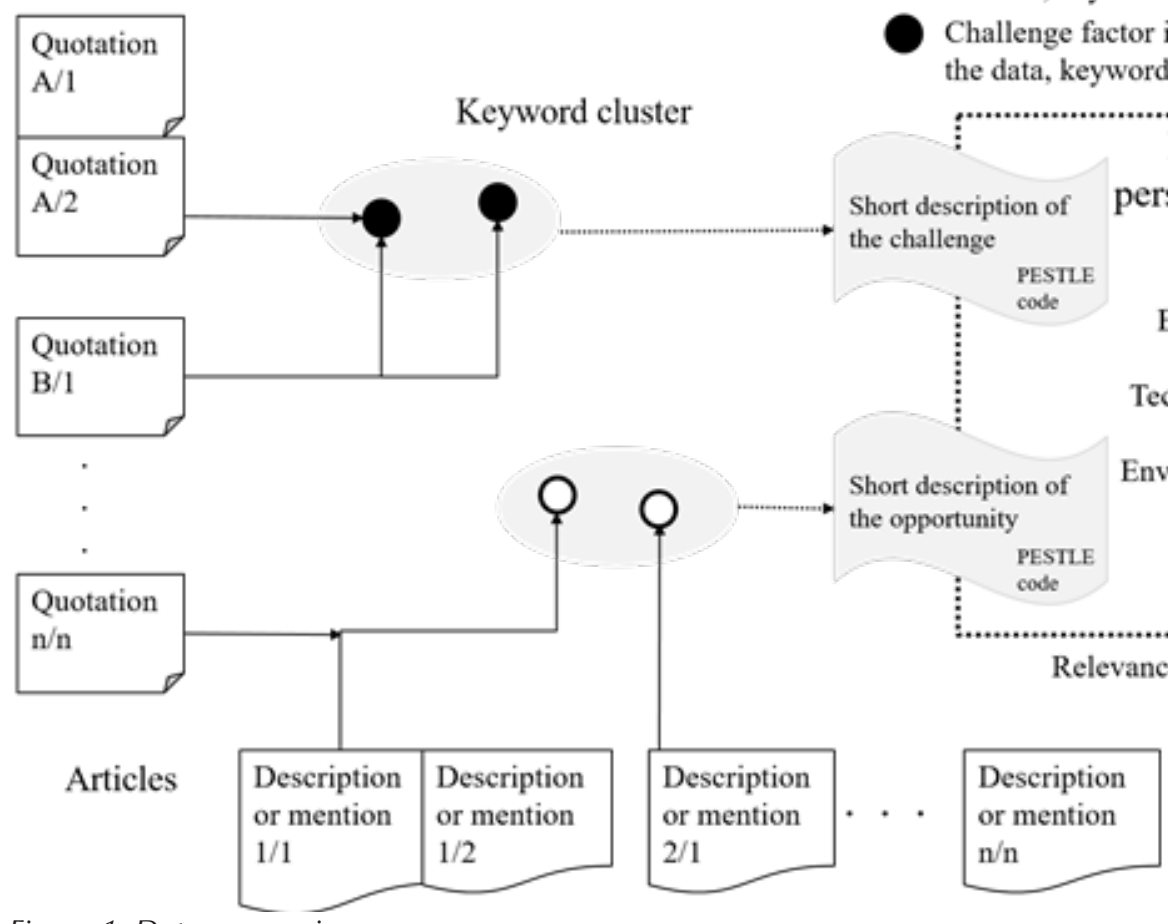

Opportunity factor identified from the data, keyword

Challenge factor identified from the data, keyword tor. The organisations of the interviewees are presented in Table 1. Thematic interviews were chosen in order to limit the discussion to topics for the interviewees to speak freely (Hirsjärvi \& Hurme, 2001). The semi-structured interviews - Acquisition and use context of Al: planning, uti- Objectives for the impact of Al utilisation: premises, opportunities, threats, risks, societal impact - Regulations and control for the use of Al: prac-

- Stakeholder identification and commitment

- Values and ethics: defining the values, ethical questions, diversity

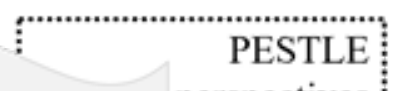

Short description of

the challenge

Technological
Legal

Technological
Legal

Economical

Social

perspectives

Political

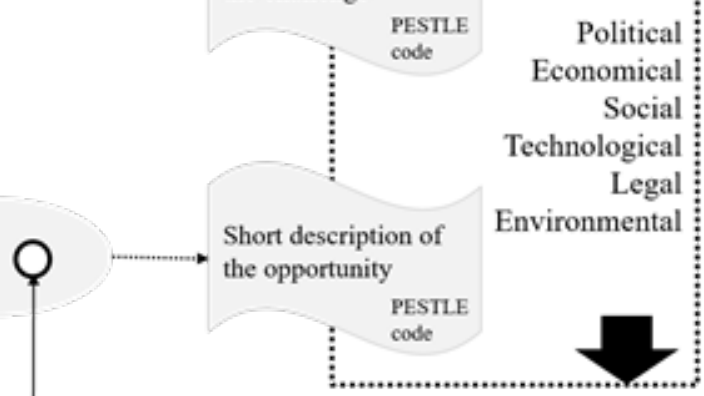

OTIPM

Figure 1. Data processing 
Table 2. The articles used in the data analysis (PQC) and the examples found (GS)

\begin{tabular}{|c|c|c|}
\hline References & Main input for $\mathrm{Al}$ in OT analysis & $\begin{array}{c}\text { Search attributes } \\
\text { PQC=ProQuest Central; GS=Google Scholar; } \\
\mathrm{Al}=\text { Artificial intelligence; OT=Occupational therapy }\end{array}$ \\
\hline AIMuhaideb et al. 2019 & $\begin{array}{l}\text { Improving communication, } \\
\text { customer appointments }\end{array}$ & PQC: 'Al' (in title) + 'physiotherapy'(in text) \\
\hline Agbo et al. 2019 & Telehealth & GS: AI, sensors, remote monitoring, rehabilitation \\
\hline André et al. 2018 & Autonomy & PQC: 'AI' (in title) + elderly (in text) + ethics (in text) \\
\hline Barros et al. 2020 & $\begin{array}{l}\text { Prevention, identification of } \\
\text { complex relations among several } \\
\text { factors }\end{array}$ & PQC: 'AI' (in title) + 'OT' (in text) \\
\hline Bhatia \& Sigh 2019 & Trends in $\mathrm{Al}$ development & PQC: 'AI' (in title) + rehabilitation (in text) \\
\hline Beil et al. 2019 & $\begin{array}{l}\text { Challenges of } \mathrm{Al} \text {-based } \\
\text { predictions }\end{array}$ & PQC: 'Al' (in title) + elderly (in text) + ethics (in text) \\
\hline Berrouiguet et al. 2019 & Utilisation of sensor data & PQC: 'Al' (in title) + rehabilitation (in text) \\
\hline Brougham \& Haar 2018 & Impact on job description & PQC: 'Al' (in title) + 'psychotherapy' (in text) \\
\hline Bublitz et al. 2019 & Opportunities of technology & PQC: 'AI' (in title) + elderly (in text) + ethics (in text) \\
\hline Carter et al. 2019 & $\begin{array}{l}\text { Bias, 'black box problem', } \\
\text { changes in conceptualisation }\end{array}$ & PQC: 'AI' (in title) + elderly (in text) + ethics (in text) \\
\hline Chakravarthy 2019 & $\begin{array}{l}\text { Preparing for the introduction of } \\
\mathrm{Al}\end{array}$ & PQC: 'Al' (in title) + rehabilitation (in text) \\
\hline
\end{tabular}

Corcoran et al. 2018 Predicting mental disorders based on machine learning

GS: Al, mental rehabilitation

Davenport et al. $2020 \quad$ Marketing perspective $\quad$ PQC: 'Al' (in title) + elderly (in text) + ethics (in text)

De Choudhury \&

Kiciman 2018

Value and threats in clinical use PQC: 'Al' (in title) + 'psychotherapy' (in text)

Dorofeev et al. 2019 of $\mathrm{Al}$

Prognostic models of spinal GS: AI, rehabilitation, models rehabilitation

Durkin 2019

Al utilisation in health sector companies

\begin{tabular}{|c|c|c|}
\hline Esfahlani et al. 2019 & $\begin{array}{l}\text { Al-based neuro-rehabilitation } \\
\text { video games }\end{array}$ & GS: AI, neurological rehabilitation \\
\hline Han et al. 2019 & Al training needs in health care & $\begin{array}{l}\text { PQC: 'AI' (in title) + elderly (in text) + ethics (in } \\
\text { text); 'Al' (in title) + 'OT' (in text) }\end{array}$ \\
\hline Huang et al. 2019 & Al in home rehabilitation & PQC: 'Al' (in title) + 'OT' (in text) \\
\hline $\begin{array}{l}\text { Jacob et al. } 2019 \\
\text { Jacob et al. } 2020\end{array}$ & $\begin{array}{l}\text { Al-based adjustable } \\
\text { compensation solutions }\end{array}$ & GS: $\mathrm{Al}$, deep learning, exoskeleton, rehabilitation \\
\hline Karches 2018 & $\begin{array}{l}\text { Effects of } \mathrm{Al} \text { on customer } \\
\text { relationship }\end{array}$ & PQC: 'AI' (in title) + elderly (in text) + ethics (in text) \\
\hline Khisamova et al. 2019 & Security risks, privacy, liability & PQC: 'Al' (in title) + rehabilitation (in text) \\
\hline Kittanawong et al. 2019 & $\begin{array}{l}\mathrm{Al} \text { in RCT research and } \\
\text { development of interventions }\end{array}$ & PQC: 'Al' (in title) + 'physiotherapy' (in text) \\
\hline Minear et al. 2019 & $\begin{array}{l}\text { Al-based chat bots in mental } \\
\text { health services }\end{array}$ & GS: $\mathrm{Al}$, chat bot \\
\hline
\end{tabular}

Simmler \& Markwalder Al-legislation and responsibility $\mathrm{PQC}$ : 'Al' (in title) + rehabilitation (in text) 2019

\begin{tabular}{lll} 
Sutrop 2019 & Liability & PQC: 'Al' (in title) + elderly (in text) + ethics (in text) \\
\hline Tran et al. 2019 & $\begin{array}{l}\text { Consideration of vulnerable } \\
\text { groups }\end{array}$ & PQC: 'Al' (in title) + elderly (in text) + ethics (in text)
\end{tabular}

Umbrico et al. $2020 \quad$ Machine learning based assistive GS: Al, rehabilitation, robot robot

Visscher et al. $2019 \quad$ Al-based diagnosis $\quad$ PQC: 'Al' (in title) + rehabilitation (in text)

\section{Zahabi \& Razak $2020 \quad$ Adaptive Al-based VR-training GS: Al, adaptive rehabilitation, virtual reality}

article and, in addition, the word pair 'occupational therapy' to appear at some point in the text. When the review was limited to peer-reviewed articles published since the beginning of 


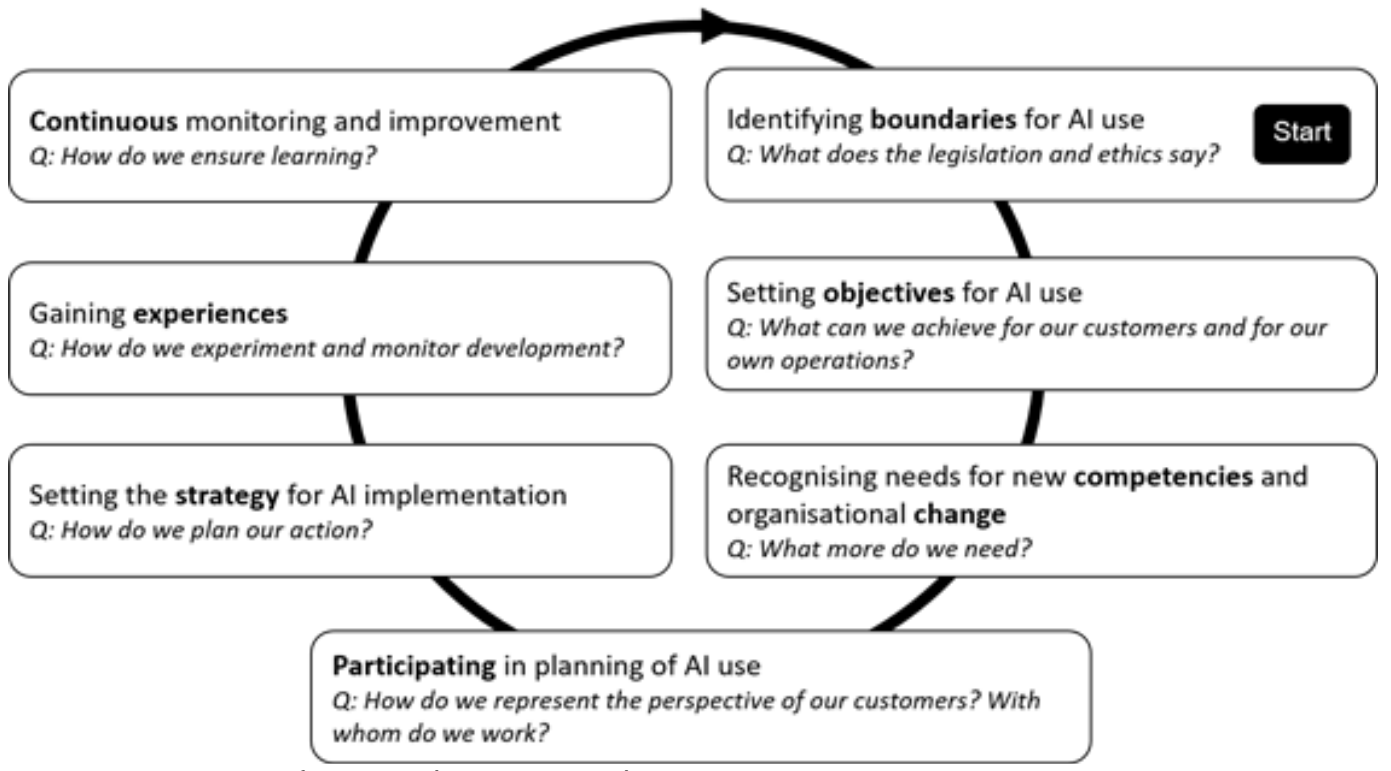

Figure 2. Basic steps for Al implementation planning

2018, three articles were found that were accessible. In addition, searches were performed using the word 'physiotherapy', 'psychotherapy' or 'rehabilitation' instead of the latter word pair OT. In addition to the above, $2 / 2$ of these articles were included in physiotherapy, 2/2 in psychotherapy, and $7 / 17$ in rehabilitation. Some of the rehabilitation articles were excluded because they did not address the utilisation of $\mathrm{Al}$ in a clinical context applicable to OT, instead of focusing purely on modelling issues or research use, for example. In order to add more home care-related knowledge we ended up, based on some test search, with the keywords 'artificial intelligence' in a title and 'elderly' and 'ethics' in the text. In this way, another 9/18 suitable articles were found. In addition, eight examples of using $\mathrm{Al}$ in therapy were found with the use of Google Scholar. All 22 articles used in the data analysis, as well as the eight examples, are presented in Table 2.

\section{Data analysis}

The interview data and the article texts were analysed using content analysis (Kohlbacher, 2006; Mayring, 2000). In adapting the technique described by Krippendorff (1980), the texts (both interview transcriptions and article text) were first divided into content areas, in this case, according to well-known PESTLE perspectives ( $\mathrm{P}=$ political, $\mathrm{E}=$ economy, $\mathrm{S}=$ social, $\mathrm{T}=$ technological, $\mathrm{L}=$ legal, $\mathrm{E}=$ environmental). PESTLE is a strategic planning tool that can be used to assess the impact of various factors on the case. The analysis is pragmatic and not intended to replace a complete and comprehensive risk analysis (e.g. Rastogi \& Trivedi 2016; Walsh et al. 2019). In this study, PESTLE analysis was used for parsing opportuni- ties and challenges identified from the data. The data processing is illustrated in Figure 1.

\section{Results}

At first, the societal and operational framework affecting OT in the background was outlined based on the PESTLE analysis. This was followed by a more detailed examination of the opportunities and challenges associated with artificial intelligence in occupational therapy. The findings are summarised in more detail in Table 3.

Opportunities and challenges are, in this chapter, separated from each other in order to illustrate factors. However, in many cases of Al, things are not black and white, but appear in different shades of grey, and/or opportunities and challenges exist at the very same time. One example of a quotation that brought out both sides of the coin, in the case of utilising a large amount of patient history data, is presented below:

"And in a way, it seems to me that this artificial intelligence, it has one side that means that it can, at best, drill-down and identify things [from the data]. But the challenge is how the matter is handled because it can become a big annoyance and worry for the person, and it can cause stigma." [D]

\section{Potential of $\mathrm{Al}$ in the rehabilitation process}

General opportunities for using $\mathrm{Al}$ in social and health care were related to a public health review based on more efficient data use and prediction models. Rationalising and developing service processes based on new information found via analysis and by automatizing certain routine work tasks, the development of diagnoses and making savings were also on the focus. 
Table 3. Summary of the main findings

Theme Short descriptions

Main opportunities:

$+\quad$ Al enables efficient use of real-time data, analytical power and prediction models to support social and health care decision-making. Possibilities for public health review and process guidance, e.g. [32]. [A, $B, D, G, H]$

+ Rationality: Al enhances action by using people's time for actions that benefit it most. Routine work automation $[A, B, C, I]$, ease and smoothness of operations $[A, G, H, I]$ performance and quality improvement $[E, F]$, patient safety $[E, H]$, partial self-service $[B, H]$, database diversification $[F, H]$. Examples include chat bots, automatic text and image recognition.

+ Service development: Al supports service uniformity, objectivity, quality, targeting, personalisation, diagnosis, e.g. [36]. [A, C, H, I]

+ Savings: Data review speeds up, cost-benefit in operation $[\mathrm{A}, \mathrm{D}], \mathrm{Al}$-based telemedicine brings about social savings, e.g. through the speed and accuracy of analysis, e.g. [42].

+ Conceptualising: Al can change understanding of social factors, assumptions about dependencies, and conceptualising illness, e.g. [33], development of diagnosis and perceptions of the disease by understanding new types of factors, e.g. [52].

Main challenges:

Societal and - Social trust: the experience of autonomy may weaken, e.g. $[27,36]$. Power structures are changing [A, operational $\mathrm{B}]$. False information or privacy concerns erode the legitimacy of actors $[\mathrm{B}]$.

framework - Privacy and individual protection are reduced, e.g. [31,32]. [C, D]

- $\quad$ Lack of skills and understanding related to AI $[A, B, D, E, F, H]$. Competence challenges in technology and legislation, as well as the lack of an $\mathrm{Al}$ strategy, limit the ethical use of Al, e.g. [39, Khi-samova et al. 2019).

- $\quad$ Lack of transparency: 'Black box problem' in use of artificial neural networks. Human cannot explain all the action and communication included, e.g. [33,37]. [A, C, G, I]

- Bias: Al prediction models may have biases related to the input data and the algorithm being taught, leading to unfair treatment of individuals and/or patient groups, e.g. [30]. These are caused by e.g. value downloads and approaches to data collection, analysis and transmission, e.g. [46] [C, D, G].

- Conflicts of values: the ethics of use cannot be guaranteed, e.g. $[7,41,45]$ usefulness vs. necessity of knowledge. [G]

- Al governance: Monitoring and managing $\mathrm{Al}$ is difficult and resource consuming $[\mathrm{A}, \mathrm{H}]$. Self-regulation is not sufficient, and it is difficult to create uniform, clear legislation on compliance with ethical principles without significantly restricting business, e.g. $[7,36][\mathrm{H}]$. Legislation updates are needed widely $[C]$, e.g. permits for real-time data use $[B, C, H]$, consent $[G]$ for automatic decision-making.

- Impact assessment is difficult. [B]

- $\quad$ Responsibility in the case of mistakes is hard to determine, e.g. [34,46,49]. [B]

Main opportunities

+ Gathering information from large databases with support of Al tools, e.g. [28] [A, C, D, F, G, H, I], use of chat bots [48].

$+\quad$ Needs and prediction: Possibility to utilise prediction models and consider complex conditional dependence relationships of variables in evaluation $[A, C, D, H, I]$. Identifying early intervention needs and targeting rehabilitation measures on an individual basis $[\mathrm{G}]$, e.g. $[28,35,37,38]$.

Evaluation + Data from monitoring performed by the customer and related parties through remote solutions can be and goalsetting phase of OTIPM (also reevaluation phase) obtained to support the observation. Al combined with wearable technology and portable mHealth solutions enables real-time data collection and analysis. Data from a variety of sources (voluntary use of technology) [F], e.g. [31,42].

Main challenges:

- $\quad$ Structural validity: The data used in the analysis does not adequately describe the issue for which it is used. The importance of context dependent situational factors that are excluded from the data-based analysis (e.g. culture, social impact, temporal factors) is not sufficiently taken into account.

- Cognitive load and usability challenges in technology use [E, F].

- Data clutter vs. format requirements and/or system structure do not allow for the use of existing data. $[C, D, G]$

- $\quad$ Experience of autonomy may weaken among customers and employees, e.g. [27]. [C, H]

In addition, conceptualising illnesses and finding new correlations were mentioned for changing perceptions and affecting new approaches. One of the interviewees expressed this as follows:
"That it can be predicted that a person who meets certain criteria is highly likely to have/be exposed to something. (...) Or it could be (...) a trend that something is emerging now, something that soci- 
Table 3. Summary of the main findings (cont.)

Theme Short descriptions

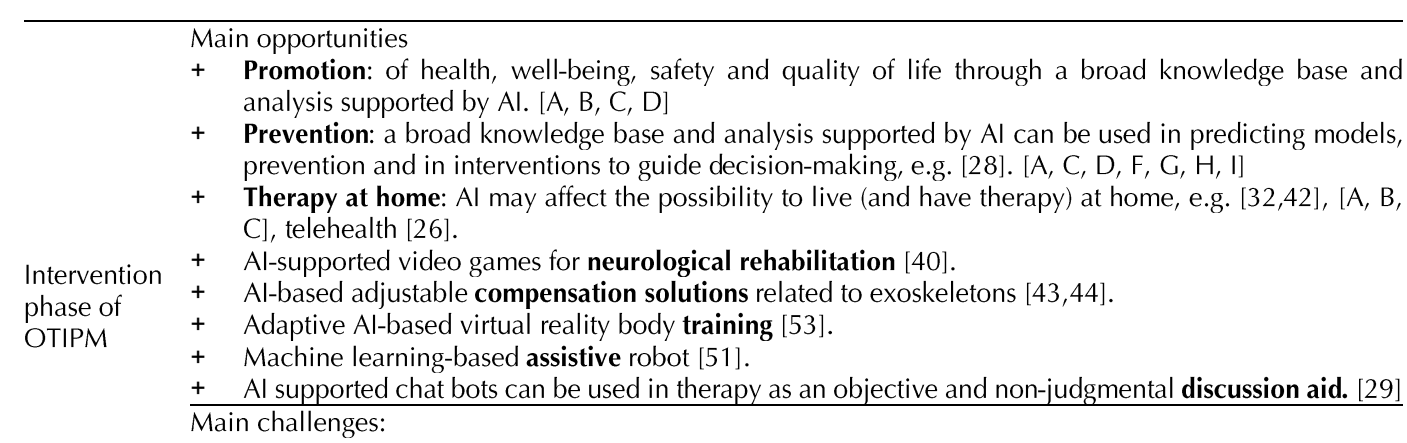

- Cognitive load and usability challenges in technology use. [E, F]

- In remote solutions, continuous monitoring and customer data collection reduces privacy. Risks related to sensitive data $[31,32]$. [C, D]

- Many of the solutions are still only for research use (not on the market), lack of practical usability [37].

\section{Main opportunities \\ $+\quad$ Customisation and personalisation. Opportunities for early interventions based on individual factors} (e.g. emotional skills, coping strategies, support networks) [31,37].

Challenges:

Customer- - Interests: The public health significance of the data to be collected and utilised overtakes the need for centric approach an individual rehabilitator. The financial interests of different actors related to the Al ecosystem may form significant conflicts with the customer's interests, e.g. [45].

- Ensuring information security is difficult and deficiencies have significant effects, e.g. [32]. Data protection requirements are challenging when personalised data is utilised $[B, C, F, H]$.

- $\quad$ The change in operating culture is large and difficult, and may fail. $[A, D, F, H]$

- The customer may not be clearly informed about the use and purpose of AI. [A, F, G, H]

- $\quad$ Some information tied to a patient's case history can be stigmatising. [C]

Main opportunities

Evidence-

based

$+\quad$ Large data sets and $\mathrm{Al}$ can be utilised in RCT studies as well as in the faster design of intervention recommendations $[28,47]$.

approach

Challenges

- The focus of data review may shift beyond clinical expertise and client insights [45].

ety has to be prepared for." $[\mathrm{H}]$

As the summary presented in Table 3 shows, gathering information from large databases with the support of Al tools was also one of the main opportunities for OTIPM. Prediction models, considerations of complex conditional dependences of variables, and identifying early intervention needs were also clear opportunities for the evaluation phase and clinical decision-making. Al-supported analysis was also perceived to contribute to the promotion of health, well-being, safety, and quality of life. In the intervention phase, Al and the development of sensor technology were thought to enable living (and having therapy) at home better than is currently the case. A few examples of Al-supported rehabilitation were found to be related to neurological rehabilitation, exoskeletons, body training, assistive robots, and chatbots (conversational $\mathrm{Al}$ ), for example.
Related to the customer-centric approach, AI was perceived to support customisation and personalisation, as well as early interventions based on individual factors (e.g. emotional skills, coping strategies, support networks). For evidencebased practice, large datasets and Al-based analysis were perceived to offer exploitation opportunities in studies and the design of intervention recommendations.

\section{Ethical challenges when utilising $\mathrm{Al}$ in the reha- bilitation process}

Using Al in social and health care contains many general challenges. Some of the most commonly discussed topics were related to social trust and the experience of autonomy, power structures, privacy concerns, transparency, and biases leading to unfair treatment of individuals and patient groups. One informant described this as follows: "Well, the threat, of course, is that data protection isn't OK, or if artificial intelligence doesn't re- 
spond to the customer's question correctly, then there will be false information; this of course it is a threat. After all, there are such horror images where information security does not work and false information goes further, so the operator loses one's legitimacy." [B]

The lack of necessary skills and understanding was perceived to hinder Al use in general. Competence challenges were recognised in technology and legislation, for example. In addition, the lack of an Al implementation strategy was perceived to limit the ethical use of AI. Conflicts between different values and difficulties in monitoring, managing, and regulating $\mathrm{Al}$ were also mentioned. The impact assessment was seen as difficult, and responsibility was hard to determine in the case of using $\mathrm{Al}$ in decision-making. One interviewee described the challenges associated with competence and utilising new knowledge produced with the help of $\mathrm{Al}$ as follows:

"First of all, it is about what these results mean to us. How do we understand these? And on the other hand, how could we utilise them then in our own preparatory work and decision-making? So it's still a kind of thing, it's been noticed that it might require a bit of a new approach. That's how to get it up and running. Compared to the fact that if we have had some individual indicators and patterns, then this is pretty different." [D]

When utilising $\mathrm{Al}$ in the evaluation phase of OTIPM, challenges should also be taken into account. The data used in the analysis should adequately describe the phenomenon for which it is used. The format requirements and/or system structure of $\mathrm{Al}$ tools do not allow the use of dishevelled data, which leads to the fact that not all customer data can be used in the analysis. However, this excluded data, as well as contextdependent situational factors (e.g. culture, social impact, temporal factors), cannot be forgotten when clinical decisions are made. The role of AI should be critically evaluated in customer work. The interviews and articles also featured discussions about the concerns related to the experience of autonomy. Both employees and customers can experience reduced autonomy when $\mathrm{Al}$ has a role in decision-making.

Cognitive load and usability challenges for personnel were also mentioned as being present when technology is used. Although we found some examples of Al-based rehabilitation methods, many of the solutions were still only for research use, and their practical usability may not be sufficient for clinical or home use. Remote solutions, continuous monitoring, and customer data collection was perceived to reduce privacy and bring about risks related to sensitive data.
One interesting question is how well the customer-centric approach can be implemented when using AI. Some doubts were presented regarding the risk that the emphasis of public health, through the data collected, may overtake the needs of the individual rehabilitator, and in addition, some other conflicts of interest between different actors in the $\mathrm{Al}$ ecosystem may also exist. As a result of this, the focus of data review (on a clinic visit) may shift beyond clinical expertise and client insights. One of the interviewees explained this:

(...) "I can't think of those people as a crowd. As a large user in volume. (...) That we are not somehow creating the kind of client-patient mass for which we are offering that "this is now the solution for everything". But, that we really could still know how to see those people as individuals and think about service from the perspective of an individual. But, then there is the dilemma that we still have to 'think big' and through whole data sets." [A]

Ensuring information security and data protection were seen as extremely important when large amounts of personalised data is utilised. Data misuse was mentioned as a risk for customers. It was also pointed out that some information tied to a patient's case history can also stigmatise the customer if it goes along with her case history, even if the information is no longer current or the data is not relevant for the purpose. Some interviewees and articles also state that if the cultural change related to $\mathrm{Al}$ use is incomplete and customers are not informed about the use and purpose of $\mathrm{Al}$, the effects are targeted at the whole service system and will have a large societal impact. The following quote illustrates the intertwining of challenges related to competence, reliability, and change in operating culture and customer understanding:

(...) "Whether the information is certainly reliable and who knows how to use it and who can or is allowed to use it, (...) That then, for the most part, we are really going there to the ethics side and thinking about the way we work. But it would be a significantly new way of acting and working. It would require a great deal of training and motivation for professionals, joint negotiation and planning, and information and communication to customers. That it is such a big upheaval..." [A]

Interviewee $\mathrm{B}$ also describes the need for change and the effects on customers as follows:

"But somehow the essential thing is that this whole idea [Al use] should get better rooted in organisations and people's core work, so that they feel it's not a mystical hype issue, but an essential information system development that benefits work and, perhaps, that also benefits customer orientation and human orientation." [B] 


\section{Discussion}

Based on the study, the key ethical challenges considering $\mathrm{Al}$ utilisation in social and health care, including rehabilitation therapy processes, are largely related to maintaining and building trust. What is especially important in terms of trust is ensuring the adequate understanding and competence of Al ethics and security, wide participation of stakeholders, and transparency in Al-based decision-making. These intertwined and wide-ranging challenges should be considered from the perspectives of society, service providers, and individual therapists and customers. All of these factors are also linked to the ethical code of conduct in OT: the pursuit of benefit, the absence of harm, autonomy, fairness, truthfulness, and reliability (AOTA 2020).

Opportunities enabled by AI for rehabilitation include clinical decision support, reducing the certain workload (for example, sophisticated natural language processing programs can transcribe, analyse, and synthesise meaning from clinical and progress notes), intervention planning, remote monitoring of home rehabilitation, and the use of Al-based rehabilitation in exercises when restoring or developing body functions.

What is needed then to materialise the opportunities and to tackle the challenges? The acquisition of $\mathrm{Al}$ requires training and cultural change to ensure these new kinds of competencies. The role of $\mathrm{Al}$ should be determined when it is used in a multi-actor and multi-disciplinary rehabilitation process. The recommendations produced by $\mathrm{Al}$ and the therapist's expertise - which emphasises the ability to make clinical decisions based on the customer's needs - together form a new way of and requirement for working. Concrete methods and the understanding of restrictions are often missed in $\mathrm{Al}$ utilisation. Al-based innovations targeted at support for acquiring and redeveloping customers' performance skills, and not just body functions, are also welcome.

To build trust, one crucial question is how different actors in the service ecosystem can act to ensure the fulfilment of responsibility and ethical values. If there are doubts about appropriateness of interests and the hidden agendas of data usage, the potential of Al may not be achieved. Further, the therapeutic relationship between the old person and the therapist requires justification for the use of Al. Because of the way Al is used, freedom of choice and protection of privacy should be clearly explained to customers. $\mathrm{Al}$ has benefits in supporting social and health care processes, but the interesting question is whether Al has the potential for the main goals of rehabilitation in terms of which are quality of life, and the experience and participation of the customer. Based on this study, this debate has not yet begun.

The utilisation of $\mathrm{Al}$ is a major societal change that affects many industries, including elderly care. No professional group creates change alone or from their separate perspectives. Instead, a wider cultural change is going on. Rehabilitation and home care professionals, need a general understanding of $\mathrm{Al}$ and its application examples. Liu (2018) reminds us that e.g, occupational therapists cannot remain as bystanders: the knowledge and holistic understanding of meaningful human activities and participation must be brought into the discussion of technology design and its use. This is in line with a LifeBased Design approach (Saariluoma, Cañas \& Leikas, 2016) that discusses ethics and highlights the need for designing for a 'good life'. It posits that the measure of technology is in its ability to enhance people's quality of life. In Figure 2, we present some basic steps and critical questions for Al implementation planning. We also suggest rehabilitation experts take part in every step and consider how these issues should be solved from the perspective of rehabilitation.

One obvious concern for $\mathrm{Al}$ use in rehabilitation is related to therapeutic rapport. The therapeutic relationship includes communication, emotional exchange, collaboration, and partnership between therapists and clients (Taylor et al., 2009). The phrase therapeutic use of self is commonly used in OT to refer to therapists' conscious efforts to establish optimal interaction with customers. Here, the therapist ponders how to bring their personality, insights, perceptions, and judgments as part of the therapeutic process (Punwar \& Peloquin, 2000). The need for empathy, which includes warmth, compassion, presence, and authenticity may not be met if there is not enough interaction between the participants in the therapy process. What happens to therapeutic rapport if Al takes on a bigger role in the rehabilitation process? Again, so far this can only be considered without experience of practical examples.

Further research is needed on how to maintain trust in the therapeutic rapport when Al applications are used. So far, there are very few practical examples of how Al changes the re-ablement process. How to build Al solutions in a way that enables an experience of inclusion, participation, and trust? One important aspect is the involvement of citizens already in the development phase of the service process. Maintaining the trust of patients and employees in the social and health care service system is a huge societal issue, and the use of $\mathrm{Al}$ adds even new dimensions to the debate. Our study confirms that more research is needed in this sector. 


\section{Conclusions}

Ethical assessment and the utilisation of $\mathrm{Al}$ in any social and health care process is highly important for preventing unexpected consequences affecting fundamental human rights. Ethics guidelines for trustworthy AI (2019) set out the key ethical principles: respect for human autonomy, prevention of harm, fairness, and explicability. These are in line with the ethical principles in rehabilitation and e.g. in OT, but the principles still leave much room for interpretation for the implementation of ethical AI.

The use of $\mathrm{Al}$ requires a balance between different efforts, innovation, safety, and efficiency. The tensions of opportunities and challenges cannot be overcome; instead, their consideration and dealing with them requires constant learning and adjustment. Rules and controls are needed, but in practice it is quite challenging to look at numerous pieces of legislation from an $\mathrm{Al}$ perspective. We have to admit that complexity is an insurmountable part of $\mathrm{Al}$. On the other hand, AI has enormous potential that can be harnessed to promote the health, well-being, safety, and quality of life of citizens and rehabilitators.

Firstly, the interviewees highlighted the challenges of competence and comprehension related to $\mathrm{Al}$, the retraining of employees, and new training programme content. Raising citizens' general awareness about $\mathrm{Al}$, the limitations of drawing conclusions based on the data, and examining the impacts of Al were also seen as being highly relevant.
Secondly, a central key theme - in Al and in therapy - is trust. Value structures and approaches related to data collection, analysis, and transmission (e.g. a lack of certain data) can produce biased data and can lead to the unfair treatment of individuals or patient groups. However, a trust includes much more, such as the transparency of data exploitation and decision-making mechanisms, and ensuring information and patient safety.

As a third broad and significant issue, the interviewees highlighted the challenges of adapting the operating culture related to Al. The change was seen to require broader policies, discussion of values and attitudes, broad citizen involvement, motivation, training for both professionals and citizens, knowledge building through trusted networks, multi-professional collaboration, communication, and sparring, for example.

Ethics discussions should take place as part of multidisciplinary dialogue, where the impacts as well as positive and negative outcomes of technology innovations are anticipated. Deliberation of the ethical impacts of $\mathrm{Al}$ in rehabilitation of older people requires consideration and debate from versatile perspectives and at different levels. This is particularly important when Al is exploited in decision-making about vulnerable older people who participate in a therapy process.

\section{Acknowledgements}

The authors wish to acknowledge the project "Ethical Al for the Governance of the Society" (ETAIROS), funded by Strategic Research Council at the Academy of Finland.

\section{References}

Agbo, C.C., Mahmoud, Q.H., \& Eklund, J.M. (2019). Blockchain technology in healthcare: a systematic review. Healthcare, 7(56)

AI HLEG. (2019). European Commission's High-Level Expert Group on Artificial Intelligence. Ethics Guidelines for Trustworthy AI. April 2019. Retrieved from https://ec.europa.eu/digital-singlemarket/en/news/ethics-guidelines-trustworthy-ai.

AlMuhaideb, S., Alswailem, O., Alsubaie, N., Ferwana, I., \& Alnajem, A. (2019). Prediction of hospital no-show appointments through artificial intelligence algorithms. Annals of Saudi Medicine, 39(6), 373-381

Alvernik, A., \& Linddahl, I. (2011). Value of occupational therapy - about evidence-based occupational therapy. Nacka: The Swedish Association of Occupational Therapists (FSA). Retrieved from https://coteceurope. eu/COTEC\%20Docs/Value\%20of\%20OT.pdf

André, Q., Carmon, Z., Wertenbroch, K., Crum, A., Douglas, F., Goldstein, W., Huber, J., van Boven,
L., Weber, B., \& Yang, H. (2018). Consumer choice and autonomy in the age of artificial intelligence and big data. Customer Needs and Solutions, 5(12), 28-37

AOTA - The American Occupational Therapy Association. (2020). The Occupational Therapy Code of Ethics. Retrieved from https://www.aota.org/ / media/Corporate/Files/Practice/Ethics/Code-ofEthics-Draft.pdf

AuroraAl (2020). National Artificial Intelligence Programme. Ministry of Finance. Retrieved from https://vm.fi/en/national-artificial-intelligence-programme-auroraai

Barros, J., Morales, S., García, A., Echávarri, O., Fischman, R., Szmulewicz, M., Moya, C., Núñez, C., \& Tomicic, A. (2020). Recognizing states of psychological vulnerability to suicidal behavior: A Bayesian network of artificial intelligence applied to a clinical sample. BMC Psychiatry, 20, 1-20

Beil, M., Proft, I., van Heerden, D., Sviri, S., \& van Heerden, P. (2019). Ethical considerations about artificial intelligence for prognostication in intensive care. Intensive Care Medicine Experimental, 7(1), 1-13

Berrouiguet, S., Barrigón, M. L., Lopez Castroman, J., Courtet, P., Artés-Rodríguez, A., \& Baca-García. E. (2019). Combining mobile-health (mHealth) and 
artificial intelligence (AI) methods to avoid suicide attempts: The Smartcrises study protocol. BMC Psychiatry, 19

Bhatia, S., \& Singh, A.Kr. (2019). Developments in artificial intelligence: a global perspective. Delhi Business Review, 20(1), 1-15

Birmingham, P. (2000). Reviewing the literature. In: D. Wilkinson (Ed.). The researcher's toolkit: the complete guide to practitioner research. London: Routledge, 25-39

Bond, R. R., Mulvenna, M. D., Finlay, D. D., \& Martin, S. (2015). Multi-faceted informatics system for digitising and streamlining the reablement care model. Journal of biomedical informatics, 56, 30-41

Brougham, D., \& Haar, J. (2018). Smart technology, artificial intelligence, robotics, and algorithms (STARA): Employees' perceptions of our future workplace. Journal of Management and Organization, 24(2), 239-257

Bublitz, F.M., Oetomo, A., Sahu, K.S., Kuang, A., Fadrique, L.X., Velmovitsky, P.E., Nobrega, R.M., \& Morita, P.P. (2019). Disruptive technologies for environment and health research: An overview of artificial intelligence, blockchain, and internet of things. International Journal of Environmental Research and Public Health, 16

Carter, S., Win, K., Wang, L., Rogers, W., Richards, B., \& Houssami, N. (2019). 65 Ethical, legal and social implications of artificial intelligence systems for screening and diagnosis. Evidence-Based Medicine, 24

Chakravarthy, M. (2019). If Oscar the cat could, can't we? - A commentary on intraoperative hypotension - Role of artificial intelligence. Indian Journal of Anaesthesia, 63(11), 875-876

Clark, H.H., \& Gerrig, R.J. (1990). Quotations as demonstrations. Language, 764-805

Cooper, H. (1998). Synthesizing Research: a Guide for Literature Reviews. Thousand Oaks: Sage Publications.

Corcoran, C.M., Carrillo, F., Fernández-Slezak, D., Bedi, G., Klim, C., Javitt, D.C., Bearden, C.E., \& Cecchi, G.A. (2018). Prediction of psychosis across protocols and risk cohorts using automated language analysis. World Psychiatry 17(1), 67-75

Davenport, T., Abhijit, G., Dhruv, G., \& Timna, B. (2020). How artificial intelligence will change the future of marketing. Journal of the Academy of Marketing Science, 48(1), 24-42

Davenport, T., \& Glover, W. (2018). Artificial intelligence and the augmentation of health care decision-making. NEJM Catalyst, 4(3)

De Choudhury, M., \& Kiciman, E. (2018). Integrating artificial and human intelligence in complex, sensitive problem domains: Experiences from mental health. Al Magazine, 39(3), 69-80

Dorofeev, N.V., Grecheneva, A.V., \& Kuzichkin, O.R. (2019). Principles of Constructing of Intellectual Systems for the Diagnostics and the Rehabilitation of the Human Spine. Procedia Computer Science, $150,270-278$

Durkin, K. (2019). Artificial intelligence-driven smart healthcare services, wearable medical devices, and body sensor networks. American Journal of Medical Research, 6(2), 37-42

EGE. (2018). European Group on Ethics in Science and
New Technologies. Statement on Artificial Intelligence, Robotics and 'Autonomous' Systems. Retrieved from https://ec.europa.eu/research/ege/pdf/ ege_ai_statement_2018.pdf.

Esfahlani, S.S., Butt, J., \& Shirvani, H. (2019). Fusion of artificial intelligence in neuro-rehabilitation video games. IEEE Access, 7, 102617-102627

Fisher, A. \& Marterella, A. (2019). Powerful practice. A model for authentic occupational therapy. Fort Collins, Colorado: CIOTS.

Floridi, L., Cowls, J., Beltrametti, M., Chatila, R., Chazerand, P., Dignum, V., Luetge, C., Madelin, R., Pagallo, U., Rossi, F, Valcke, P., \& Vayena, E. (2018). Al4People - An Ethical Framework for a Good AI Society. Minds Mach. 28, 689-707

Glendinning, C., Jones, K., Baxter, K., Rabiee, P., Curtis, L.A., Wilde, A., Arksey, H., \& Forder, J.E. (2010). Home Care Re-ablement Services: Investigating the longer-term impacts. Working Paper No. DHR 2438, University of York.

Glueck, D. (2013). Establishing therapeutic rapport in telemental health. In: D. Glueck, K. Myers \& C.L. Turvey (Eds.), Telemental health: Clinical, technical and administrative foundations for evidence-based practice, 29-46.

Government Artificial Intelligence Readiness Index 2019 (2019). Oxford Insights. Retrieved from https://www.oxfordinsights.com/ai-readiness2019

Han, E., Yeo, S., Min-Jeong, K., Young-Hee, L., Park, K., \& Roh, H. (2019). Medical education trends for future physicians in the era of advanced technology and artificial intelligence: An integrative review. BMC Medical Education, 19, 1-15

Hirsjärvi, S. \& Hurme, H. (2001). Tutkimushaastattelu - Teemahaastattelun teoria ja käytäntö. Helsinki: Yliopistopaino. (In Finnish.)

Huang, G., Yu-Zhou, L., \& Zhi-Wang, Q. (2019). Critical factors in the application of artificial intelligence to establish health care systems for seniors. Revista De Cercetare Si Interventie Sociala, 65, 51-59

Sunil, J., Mukil, A., Varun, M.G, Manoj, K.B, Noor, Z., Vasaki, P., Shynu, P.G., \& Venki, B. (2020). An adaptive and flexible brain energized full body exoskeleton with IoT edge for assisting the paralyzed patients. IEEE Access, 8, 100721-100731

Sunil, J., Varun, M.G, Al-Turjman, F., Vinoj, PG , \& Mostarda, L.L. (2019). Artificial muscle intelligence system with deep learning for post-stroke assistance and rehabilitation. IEEE Access, 7, 133463-133473.

Karches, K.E. (2018). Against the iDoctor: Why artificial intelligence should not replace physician judgment. Theoretical Medicine and Bioethics, 39(2), 91-110

Khisamova, Z.I., Begishev, I.R., \& Sidorenko, E.L. (2019). Artificial intelligence and problems of ensuring cyber security. International Journal of Cyber Criminology, 13(2), 564-577

Kitchin, R. (2017). Thinking critically about and researching algorithms. Information, Communication \& Society, 20(1), 14-29.

Krittanawong, C., Johnson, K.W., \& Tang, W.H. (2019). How artificial intelligence could redefine clinical trials in cardiovascular medicine: Lessons learned from oncology. Personalized Medicine, 16(2), 87-92

Kohlbacher, F. (2006). The use of qualitative content 
analysis in case study research. Forum of qualitative social research. Retrieved from http://www. qualitative-research.net.

Krippendorff, K. (1980). Content analysis: An introduction to its methodology. London, Sage.

Siddique, L., Rajib, R., Junaid, Q., Anwaar, A., Muhammad, A.I., \& Muhammad, S.Y. (2017). Mobile health in the developing world: Review of literature and lessons from a case study. IEEE Access, 5, 11540-11556

Leikas, J., Sigfrids, A., Stenvall, J., \& Nieminen, M. (2020). Good Life Ecosystems - Ethics and Responsibility in the Silver Market. In: Rauterberg M. (Ed.) Culture and Computing. HCII 2020. Lecture Notes in Computer Science, vol 12215. Springer, Cham, pp. 105122 https://doi.org/10.1007/978-3-030-50267-6_9

Liu, L. (2018). Occupational therapy in the fourth industrial revolution. Canadian Journal of Occupational Therapy, 85(4), 272-285

Maddox, T.M., Rumsfeld, J.S., \& Payne, P.R. (2019). Questions for artificial intelligence in health care. Journal of American Medical Association (JAMA), 321(1), 31-32

Mayring, P. (2000). Qualitative content analysis. FQS Forum: Qualitative social research. Retrieved from http://www.ualitative-research.net/fqs/.

Miner, A.S., Shah, N., Bullock, K.D., Arnow, B.A., Bailenson, J., \& Hancock, J. (2019). Key considerations for incorporating conversational $\mathrm{Al}$ in psychotherapy. Frontiers in Psychiatry, 10:746. https://doi. org/10.3389/fpsyt.2019.00746

Petch, A. (2008). Re-ablement and the role of the occupational therapist, Journal of Integrated Care, 16, 2, 38-39

Punwar, J. \& Peloquin, M. (2000). Occupational therapy: Principles and practice. Philadelphia: Lippincott.

Rajkomar, A., Dean, J., \& Kohane, I. (2019). Machine learning in medicine. New England Journal of Medicine, 380(14), 1347-1358

Rastogi, N., \& Trivedi, M.K. (2016). PESTLE technique - a tool to identify external risks in construction projects. International Research Journal of Engineering and Technology, 3(1), 384-388

Russell, S.J. \& Norvig, P. (2021). Artificial Intelligence - A Modern Approach. Fourth Edition. Harlow: Pearson Education. Retrieved from http://aima. cs.berkeley.edu/

Saariluoma, P., Cañas, J.J. \& Leikas, J. (2016). Designing for Life - A human perspective on technology development. London: Palgrave MacMillan. https:// doi.org/10.1057/978-1-137-53047-9

Sackett, D.L., Rosenberg, W.M.C., Gray, J.A.M., Haynes, R.B., \& Richardson, W.S. (1996). Evidence-Based Medicine: What it is and what it isn't. British Medical Journal, 312, 71-2

Simmler, M., \& Markwalder, N. (2019). Guilty robots? - rethinking the nature of culpability and legal per- sonhood in an age of artificial intelligence. Criminal Law Forum, 30(1), 1-31

Stead, W.W. (2018). Clinical implications and challenges of artificial intelligence and deep learning. Journal of American Medical Association (JAMA), 320(11), 1107-1108

Sutrop, M. (2019). Should we trust artificial intelligence? A Journal of the Humanities and Social Sciences, 23(4), 499-522

Taylor, R.R., Lee, S.W., Kielhofner, G., \& Ketkar, M. (2009). Therapeutic use of self: A nationwide survey of practitioners' attitudes and experiences. American Journal of Occupational Therapy, 63(2), 198-207

Tran, B.X., Mclntyre, R.S., Latkin C.A., Phan, H.T., Vu, G.T., Nguyen, H.L.T, Gwee, K.K., Ho, C.S.H., \& Ho, R.C.M. (2019). The current research landscape on the artificial intelligence application in the management of depressive disorders: A bibliometric analysis. International Journal of Environmental Research and Public Health, 16, 2150

Tuominen, H. (2019). Tekoälyn perusteita. In: P. Neittaanmäki (Ed.), H. Tuominen, S. Äyrämö \& P. Vähäkainu. Tekoäly ja terveydenhuolto Suomessa [Artificial Intelligence and Health Care in Finland]. Jyväskylä: Jyväskylän yliopisto, 11-26. (In Finnish.)

Umbrico, A., Sorrentino, A., Cavallo, F., Fiorini, L., Orlandini, A. \& Cesta, A. (2019). Toward the Integration of Perception and Knowledge Reasoning: An Adaptive Rehabilitation Scenario. 5th Italian Workshop on Artificial Intelligence for Ambient Assisted Living, AI*AAL.it 2019, Rende, Italia, 20.11.2019. Retrieved from http://ceur-ws.org/Vol-2559/paper1.pdf

Vaidyam, A.N., Wisniewski, H., Halamka, J.D., Kashavan, M.S., \& Torous, J.B. (2019). Chatbots and conversational agents in mental health: a review of the psychiatric landscape. The Canadian Journal of Psychiatry, 64(7), 456-464

Visscher, R.M.S., Feddermann-Demont, N., Straumann, Romano, F., Straumann, D., \& Bertolini, G. (2019). Artificial intelligence for understanding concussion: Retrospective cluster analysis on the balance and vestibular diagnostic data of concussion patients. PLoS One, 14(4): e0214525. https://doi. org/10.1371/journal.pone.0214525.

Wade, E., \& H Clark, H.H. (1993). Reproduction and demonstration in quotations. Journal of Memory and Language, 32(6), 805-819

Walsh, K., Bhagavatheeswaran, L., \& Roma, E. (2019). E-learning in healthcare professional education: an analysis of political, economic, social, technological, legal and environmental (PESTLE) factors. MedEdPublish, 8, https://doi.org/10.15694/ mep.2019.000097.1

Zahabi, M., \& Razak, A.M.A. (2020). Adaptive virtual reality-based training: a systematic literature review and framework. Virtual Reality, 725-752 